\title{
Erratum: "Acoustic mode radiation from the termination of a truncated nonlinear internal gravity wave duct in a shallow ocean area" [J. Acoust. Soc. Am. 126, 1752-1765 (2009)]
}

\author{
Ying-Tsong Lin, Timothy F. Duda, and James F. Lynch \\ Department of Applied Ocean Physics and Engineering, Woods Hole Oceanographic Institution, Woods \\ Hole, Massachusetts 02543
}

(Received 23 January 2010; revised 26 April 2010; accepted 3 May 2010)

[DOI: $10.1121 / 1.3436545]$

PACS number(s): 43.30.Re, 43.20.Bi, 43.20.Mv, 43.30.Bp [JAC]

In Eq. (10c) a typographical error involving a missing equality symbol occurred. The correct expression is as follows:

$$
\begin{aligned}
\Phi_{n m}(y)= & {\left[e^{-i \sqrt{\zeta_{m}^{2}-\kappa_{n m}^{2} y}}+R_{L m} e^{i \sqrt{\zeta_{m}^{2}-\kappa_{n m}^{2}}(y+D)}\right] } \\
& \text { for } \quad-D / 2 \leq y \leq D / 2 .
\end{aligned}
$$

In addition there is an error with Eq. (13), and this equation should read

$$
\begin{aligned}
\Gamma_{m}(x, y)= & -\pi \frac{\Psi_{m}\left(x_{S}, y_{S}, z_{S}\right)}{\rho\left(z_{S}\right)} \zeta_{m} \sum_{n} \frac{\Phi_{n m}\left(y_{S}\right)}{\kappa_{n m} w_{n m}} e^{i \kappa_{n m}\left(L-x_{S}\right)} \\
& \times \int_{-\infty}^{\infty} \Phi_{n m}(\eta) \frac{x-L}{\sqrt{(x-L)^{2}+(y-\eta)^{2}}} \\
& \times H_{1}^{(1)}\left(\zeta_{m} \sqrt{(x-L)^{2}+(y-\eta)^{2}}\right) d \eta .
\end{aligned}
$$

These two errors have been corrected in the electronic copy stored in the ASA Digital Library. 